Meta

Journal des traducteurs

Translators' Journal

\title{
La langue française de l'informatique envisagée depuis une perspective américaine
}

\section{Élisabeth Eek}

Volume 43, numéro 3, septembre 1998

URI : https://id.erudit.org/iderudit/002698ar

DOI : https://doi.org/10.7202/002698ar

Aller au sommaire du numéro

Éditeur(s)

Les Presses de l'Université de Montréal

ISSN

0026-0452 (imprimé)

1492-1421 (numérique)

Découvrir la revue

Citer cet article

Eek, É. (1998). La langue française de l'informatique envisagée depuis une perspective américaine. Meta, 43(3), 455-462. https://doi.org/10.7202/002698ar
Résumé de l'article

Le français de l'informatique peut être, d'une part, envisagé depuis différents horizons (France, États-Unis) et également englober différentes logiques linguistiques. D'autre part, une analyse des transferts terminologiques va déclencher, à partir du principe de "mouvance" des idées, une remise en cause du concept traditionnel d'emprunt. Il est bon de rappeler que les terminologies mises au point par les commissions d'experts doivent reposer sur une sémantique reconnaissable par la majorité des locuteurs. L'ignorance de ce principe risque d'engendrer un rejet des nouvelles terminologies, en particulier par les spécialistes de l'informatique eux-mêmes, dont l'attitude de "malaise" linguistique vaut quelques explications. Il conviendra également de rappeler l'influence du français de l'informatique sur l'anglais de même que l'existence des industries de la langue, nécessaires pour faire du fran- çais une langue véhiculaire de la science.
Tous droits réservés @ Les Presses de l'Université de Montréal, 1998
Ce document est protégé par la loi sur le droit d'auteur. L'utilisation des services d'Érudit (y compris la reproduction) est assujettie à sa politique d'utilisation que vous pouvez consulter en ligne.

https://apropos.erudit.org/fr/usagers/politique-dutilisation/ 


\section{BLOC-NOTES}

\section{LA LANGUE FRANCAISE DE L'INFORMATIQUE ENVISAGÉE DEPUIS UNE PERSPECTIVE AMÉRICAINE}

\section{Résumé}

Le français de l'informatique peut être, d'une part, envisagé depuis différents horizons (France, États-Unis) et également englober différentes logiques linguistiques. D'autre part, une analyse des transferts terminologiques va déclencher, à partir du principe de «mouvance» des idées, une remise en cause $d u$ concept traditionnel d'emprunt. Il est bon de rappeler que les terminologies mises au point par les commissions d'experts doivent reposer sur une sémantique reconnaissable par la majorité des locuteurs. L'ignorance de ce principe risque d'engendrer un rejet des nouvelles terminologies, en particulier par les spécialistes de l'informatique eux-mêmes, dont l'attitude de «malaise» linguistique vaut quelques explications. Il conviendra également de rappeler l'influence du français de l'informatique sur l'anglais de même que l'existence des industries de la langue, nécessaires pour faire du français une langue véhiculaire de la science.

\section{Abstract}

This article looks at the use of the French language in the area of computer science. The author points out that French offers numerous lexical resources for expressing ideas and concepts just as effectively as English, and seeks to debunk the myth that French is less effective when it comes to expressing scientific ideas.

Le français du langage informatique peut certainement faire l'objet de nombreuses études envisagées sous différents angles, selon que l'on se place d'un point de vue purement «hexagonal» ou bien «exilé», tel qu'exprimé par les locuteurs français vivant sous l'influence intégrale d'une autre langue - et quelle langue ! Celle qui se trouve à l'origine de la polémique sévissant en France entre les puristes du français et les laxistes du vocabulaire (souhaitons que la syntaxe, considérée par R. de Gourmont ${ }^{1}$ comme l'épine dorsale du langage, soit encore épargnée).

Des enjeux sérieux sont à considérer, parmi lesquels la valeur sémantique des emprunts faits indûment à l'anglais en raison de l'ignorance académique de cette dernière langue par les locuteurs français, emprunts qui peuvent dénaturer le concept derrière le mot et, par là même, promouvoir une confusion dans la communication orale et écrite.

Ce trait explique la persistance d'une attitude fondée davantage sur une mode entrainée par les médias que par une connaissance scolaire, dont toute trace, apparemment, a disparu de la mémoire des emprunteurs... On pourrait peut-être tenter d'attribuer ce phénomène à la pénurie d'enseignements linguistiques qui permettraient aux intéressés d'analyser, derrière le lexique anglo-américain emprunté, la véracité des concepts ${ }^{2}$.

Dans cette philosophie, et à l'occasion d'une thèse patronnée par le département des langues et littératures étrangères à l'Université George-Mason (Virginie, USA), il a été jugé nécessaire de passer au crible le vocabulaire informatique utilisé en France pour réaliser combien la sémantique est malmenée par les spécialistes de l'informatique, en particulier les praticiens qui, il est regrettable de le dire, enveloppent leur art d'une atmosphère de «secte» tendant à créer un jargon pour «initiés» seulement. Cet art, qu'ils ont l'air de considérer comme «leur» chasse gardée des sciences de l'information, va tendre à se populariser malgré eux. Ce phénomène, aisément prévisible depuis l'apparition des interfaces logicielles personnelles, se confirme si l'on en juge par les réactions du public après le lancement sur le marché du système Windows 95.

Cette recherche, envisagée d'après une optique américaine, a tendance à se démarquer des études plus conventionnelles en ce qu'elle bouscule — à plusieurs égards - les conceptions traditionnelles de l'emprunt de même qu'elle introduit une nouvelle notion, les «xéno-pérégrinismes» ${ }^{3}$. De plus, son champ d'investigation recouvrira de manière synthétique la sphère la plus vaste possible des formes d'expression informatique, depuis la théorie jusqu'aux logiciels en passant par les journaux professionnels et le jargon des praticiens.

Elle rend également hommage à l'imagination lexicale des créateurs de logiciels; rétablit la vérité sémantique derrière des emprunts de mauvais aloi et, enfin, tentera d'analyser les raisons de l'attitude des locuteurs français, qui se distingue clairement de celle de leurs homologues québécois: en effet, ceux de l'Hexagone ne se sentant au départ nullement «cernés» par l'anglais auraient tendance à traiter avec légèreté la langue française, pensant qu'il est plus facile d'importer des mots anglais que de créer des gallicismes en

Meta, XLIII, 3, 1998 
raison d'une ignorance marquée des possibilités lexicales et traductives du français. L'on pourrait, de ce fait, attribuer ces lacunes à une défaillance de l'enseignement du français en France. Manifestant dès lors une perte de confiance dans leur patrimoine culturel, il n'est pas étonnant que les scientifiques français aillent au plus pressé en imitant avec maladresse l'anglais correspondant à leur domaine d'activités. Il est bon, cependant, de ne pas se laisser impressionner par les mauvais augures présageant que le français va s'étioler du fait du nombre d'emprunts à l'anglais (relevés dans les moyens d'expression modernes, fussent-ils dans la presse grand public ou les sciences de l'information). Si l'on examine la situation en face, l'on se rendra compte que l'enseignement du français sous la précédente administration a effectivement pris de la gîte ${ }^{4}$, en raison de préjugés vers le «technologisme»", embrassant une conception erronée de la culture moderne : croire que «moderniser» une société signifie plaquer sur la langue populaire des lexies étrangères en faisant fi des ressources lexicales indigènes. La modernisation d'une culture n'a jamais signifié éradiquer la langue nationale : cela signifie non seulement rénover l'outil de production matériel, mais également humain, en renforçant l'enseignement des disciplines scientifiques et techniques, à quoi nous ajouterons la nécessité d'aménager le lexique en l'équipant de termes adéquats pour désigner les nouveaux concepts mis au point par les chercheurs français ${ }^{6}$.

\section{NOUVEAUTÉS LEXICALES}

Mais la lecture des différents ouvrages étudiés ${ }^{7}$ témoigne d'un phénomène inattendu : en effet, ceux-ci font preuve d'une richesse linguistique remarquable en dépit d'une influence marquée de la langue anglaise sur certains concepts, ceux, naturellement, qui ont été découverts aux États-Unis. Hormis cette situation, qui s'apparente à celle des technologies françaises ayant donné le ton à un vocabulaire universellement utilisé (l'on peut penser aux industries des transports ferroviaires par exemple), nous observerons, de la part des théoriciens étudiés, une indéniable habileté à faire usage des facultés dérivatrices et néologiques que recèle le «génie» de la langue. À cela s'ajoutent une évidente flexibilité du lexique chez les journalistes de la presse spécialisée et une relative confiance en soi chez les concepteurs ${ }^{8}$ de deux logiciels passés en revue.

Ainsi, depuis les extensions de sens (organoleptique) jusqu'aux néologismes (traçabilité) en passant par les nombreux calques littéraux, l'on se rend compte que le stock lexical français se prête, par transparence symbolique, à l'élaboration d'une terminologie moderne. Quant aux calques sémantiques, dont l'usage est de bonne guerre lorsque les circonstances le permettent, ils ne présentent pas de problème de légitimité, puisque leur enveloppe existait déjà en français. Que le sens (ou leurs sens) en ait été changé sous la pression des événements technologiques n'importe pas tant que le signe était préexistant. Il s'agit simplement de l'adapter. Cela prouve que les ressources lexicales sont présentes pour faire face à l'assaut terminologique anglo-américain. Les exemples abondent dans les textes étudiés. L'on peut relever parmi ces ressources des extensions de sens: presse-papiers, désactivée, racine; des néologismes: déprotéger, géocodage, désinstaller, rémanence, raccourci clavier, l'ensemble constituant des atouts facilitant la communication technique, ce qui est après tout l'objectif des entreprises d'aménagement linguistique destinées à faire du français une langue de communication moderne. Rappelons les propos de Jean Dutourd, de l'Académie française, parlant de l'obligation faite aux savants français de faire leur communication dans leur langue maternelle : «Ils répondront que le reste du monde ne les lirait pas. À quoi je rétorque que si leurs découvertes sont importantes, le reste du monde se donnera la peine de les traduire» ${ }^{9}$. Cela n'interdit pas d'aménager une langue pour la rendre encore plus adéquate aux besoins de communication rapide qui caractérisent notre époque. Or, le fonds gréco-latin que le français et l'anglais possèdent en commun facilite la tâche ${ }^{10}$ : il suffit de puiser dans la technique de construction d'affixes pour altérer et redéfinir le sens derrière les mots.

En revanche, c'est dans l'attitude des praticiens de l'informatique que le bât blesse. L'on détectera chez cette communauté une impression d'exclusivité qui n'a pas de raison d'être si l'on prend conscience du nombre de bévues sémantiques dont elle se rend coupable. En effet, faussement informés quant à la valeur conceptuelle servant de fondement à leur vocabulaire, ces locuteurs vont propager, d'erreurs en préjugés, une «culture» faite d'idées préconçues et élitistes d'un autre âge. Comme le démontre l'enquête de le GuillyWallis ${ }^{11}$, il est temps de démystifier le langage informatique français et de le dépouiller des emprunts intempestifs qui peuvent fort bien être remplacés soit par leur équivalent français lorsqu'ils existent, soit par des adaptations calquées, néologiques ou dérivées, mais puisant dans le stock lexical même qui a permis la constitution de gallicismes.

Dans ce contexte, il est bon de dissiper de fausses conceptions : il ne s'agit pas de confondre le phénomène de l'emprunt, où les locuteurs ont le choix de moyens d'expression entre plusieurs langues, avec une situation de suprématie commerciale engendrant une condition de diktat linguistique : en effet, les locuteurs dans ce cas sont confrontés à une offre sans alternative, d'un médium linguistique imposé par le vendeur $^{12}$. Les acheteurs se sentent alors contraints de s'y plier.

(Ce dilemme ressort clairement de l'interview du groupe de travail Le Guilly-Wallis, mentionné plus haut.)

En revanche, il fut noté dans l'un des ouvrages étudiés l'apparition de termes non classifiables dans les catégories linguistiques connues jusqu'à ce jour : l'on se réfère ici à ce que nous baptiserons des «xénopérégrinismes», provenant de la nature hybride de 
lexies utilisées par l'auteur passé en revue. Ce ne sont en effet ni des pérégrinismes, ni des xénismes, ni de véritables emprunts : du fait de leurs traits particuliers (caractère éphémère, absence de signes métalinguistiques et multiplicité lexicale - à l'inverse des xénismes définis dans le dictionnaire linguistique de Dubois), il a fallu leur donner une définition qui correspondît à leur véritable nature. Ainsi, les instructions anglaises que l'on trouve dans le texte en question étant en général d'ordre temporaire, l'on peut tabler sur le fait que la génération suivante de manuels d'informatique $^{13}$ risque de ne plus mentionner ces mêmes instructions en anglais, mais au contraire de les présenter en français, avec parfois introduction de leur équivalent en anglais ${ }^{14}$.

Nous citerons par exemple :

CREATE INDEX, ou DROP INDEX ${ }^{15}$ : l'administrateur de données peut créer ou supprimer un index à tout moment, sans mettre en danger les programmes existants.

BEGIN (ou) END TRANSACTION : comme leur aspect l'indique, ces termes signalent le début ou la fin d'une transaction. Ils sont souvent utilisés en «chapeau» autour d'autres instructions machines plus particulières telles que celles rencontrées dans un programme écrit par l'équipe d'informaticiens du magazine Ordinateur 01.

EXPLAIN : instruction dont le rôle est de fournir avec rigueur le format interne du plan d'exécution d'un programme.

Le sort de ces termes quant à leur intégration dans le stock lexical français dépendra du niveau de culture linguistique des utilisateurs et de la bonne santé de la recherche informatique en France, capable à long terme d'engendrer plus de mots d'allure française.

$\mathrm{Si}$, par exemple, l'auteur en question se contentait de reproduire les instructions - en anglais - des machines et systèmes qu'il étudie, nous serions dans une situation critique. De mauvaises langues seraient prêtes à sonner le glas de la langue informatique française (nous disons bien «informatique», non pas le vocabulaire courant).

Mais là n'est pas le cas : cet auteur explicite en français les mécanismes opérés par la plupart des machines et systèmes vendus sur le marché. Puis il indique par quel vocabulaire ils sont exprimés dans l'anglais connu des informaticiens.

Les instructions en anglais sont mises au service des démonstrations faites en français, dont les illustrations de cas particuliers sont également faites dans la langue de Descartes.

Les instructions attachées aux systèmes (comprenant matériels et logiciels), que l'auteur explique, constituent donc des «traces» de la langue anglaise dans la mesure où l'industrie et l'enseignement informatiques en France se sont développés durant les vingt dernières années en une science authentique, avec bien sûr ses emprunts à l'anglais pour ce qui a été intégré au vocabulaire français, mais aussi des trouvailles pour le reste, telles que «jointure» ${ }^{16}$, employé dans l'ouvrage étudié. Plus important, l'entière gram- maire demeure française, ce qui est essentiel, l'apport d'emprunts ayant perdu leur allure anglaise ne faisant qu'enrichir le vocabulaire qui se francisera peu à peu, tel «parking» dans le langage courant, qui à l'heure actuelle ne «sent» plus son anglais et a été mis au service de la communauté linguistique française.

\section{REMISE EN CAUSE DE L'EMPRUNT}

Inversement, nous avons rencontré d'indéniables emprunts, sous la forme d'unités lexicales (clusters, par ex.) ou de conventions de l'industrie informatique (SQL, B-TREE). Mais, comme nous l'avons observé plus haut, la majorité des désignations relevées dans les textes étudiés est faite de termes en provenance du stock lexical français, soit sous forme d'extensions et de calques, soit de dérivés et néologismes; la syntaxe demeure, malgré quelques rares impairs (de nature bénigne), quasiment irréprochable.

Ces remarques pourraient éventuellement nous permettre de remettre en cause le principe de l'emprunt. 11 semblerait en effet que ce concept traditionnel ne soutienne pas l'examen linguistique contemporain : si les définitions que l'on trouve chez Dubois et Chansou postulent que le concept et le signe (à la fois) sont ${ }^{17}$ transférés dans le patrimoine linguistique emprunteur, cela est une approche statique qui ne correspond pas à la réalité technique contemporaine où l'évolution, le déplacement des idées priment sur la fixité attachée aux phénomènes linguistiques traditionnellement appréhendés.

En effet, un concept mis au point par une communauté linguistique quelconque va engendrer la création ou l'adaptation de signes pour l'exprimer. Par voie de conséquence, la mode et les médias vont propager telle une onde de choc cette nouvelle expression, qui va engendrer à son tour soit son acceptation d'abord pure et simple par la communauté emprunteuse, en attendant qu'elle soit transformée plus tard, soit sa traduction immédiate en un lexème qui «sente» la langue du terroir, tel que la puce pour le micro chip ou convivial pour user friendly ou informatique pour computer science.

Mais les concepts qui se trouvent derrière les nouvelles dénominations appartiennent à l'humanité entière; seule leur expression fait l'objet, tout d'abord, de la protection légale accordée aux brevets et droits d'auteurs. Lorsque ces dénominations tombent dans le domaine public, elles ne sont plus protégées. C'est ainsi que le concept et l'idée des $« \mathrm{SGDB}{ }^{18}$ n'appartiennent pas à E. F. Codd, ingénieur chez IBM qui les a mises au point. Elles constituent des conventions techniques dont tout fabricant de système de gestion de données se sert à l'heure actuelle. Seule leur expression relève encore du domaine protégé des termes onomastiques, et ce jusqu'à ce qu'elles tombent dans le domaine public.

Citons sur ce point le propos de M. Kahn, à l'époque président de Borland, s'adressant à M. Manzi, président de Lotus : «You knew, like all of us, that 'system' 
and 'functionality' cannot be copyrighted ${ }^{19}$. Seule, donc, est protégée la version particulière de chacun des fabricants qui adapte ces systèmes à sa clientèle. C'est là tout l'intérêt de la législation sur les brevets et les droits d'auteur, l'alternative conduisant à l'étouffement de la créativité humaine. C'est précisément pour permettre l'échange d'idées nécessaires au progrès que la législation sur les droits d'auteur et brevets a prévu la protection de l'expression seulement et non du concept à la base de cette expression.

C'est pourquoi l'agitation faite il y a quelques années autour de la stratégie japonaise qui consistait à emprunter des concepts mis au point aux États-Unis ou ailleurs et de les revêtir d'une griffe «made in Japan», après les avoir modifiés ou améliorés, n'a pas de sens, car ces concepts n'ont jamais appartenu à quiconque, seule leur expression pouvait être jalousement gardée, et cela uniquement jusqu'à l'expiration de leur protection légale. Ce qui nous amène à nous demander en fonction de quel critère le comité linguistique considère ${ }^{20}$ qu'un concept est «emprunté».

Par principe, une idée appartient à l'humanité entière. Que l'un quelconque de ses locuteurs ait su l'exprimer à sa façon n'importe pas. C'est ainsi que tout le monde a pu exploiter le mécanisme de la boîte de vitesses automatique sans se soucier de qui en a déposé le brevet le premier, l'important étant de le mettre au point de manière à le rendre immédiatement utilisable par la communauté intéressée, dans la mesure, naturellement, où le brevet de fabrication en fut protégé. Mais le concept lui-même peut être exploité par quiconque trouve le moyen de l'exprimer en lui imprimant les marques de sa propre ingénuité. Ces marques seules sont protégées et non le concept les soutenant.

Nous devrons approuver par conséquent la position de François Gaudin, qui adhère à la notion de «nomadisme» des idées mise au point par les philosophes des sciences ${ }^{21}$.

Partant de ce raisonnement, nous ne voyons pas pourquoi un emprunt serait à la fois l'enveloppe lexicale d'un mot et son signifié. L'emprunt ne devrait être qu'un signifiant. Ce qui revient à dire que tant que le stock lexical français sera capable de fournir une réponse de quelque forme que ce soit (néologisme, calque, dérivé, etc.) au défi linguistique anglo-américain et que cette forme revêtira l'aspect d'un gallicisme tel que la puce ou le bogue (pour bug), la langue française sera loin de s'étioler, n'en déplaise à un scientifique interrogé par F. Gaudin dans Meta ${ }^{22}$. Naturellement, il est judicieux pour toute communauté linguistique de mettre au point un concept scientifique afin d'en inventer le support terminologique et de le répandre alentour (nous pourrions faire allusion, pour ce qui est des Français, non seulement à la sphère biologique avec la pasteurisation, mais également au domaine des seuils de réfrigération des supraconducteurs $^{23}$, pour ne citer que quelques exemples). Mais c'est également faire preuve de bonne santé linguistique que de pouvoir puiser dans son réservoir lexical (et les ressources dérivatives de sa propre langue) le matériau permettant de transformer un lexème importé en un signifiant qui ressemble à un gallicisme. Qu'importe donc si les concepts ont été découverts par une communauté $\mathrm{X}$ lorsque la communauté Y peut les habiller d'un signe qui se moule dans la tradition linguistique propre à cette dernière catégorie de population?

Nous ne voyons point, par conséquent, le danger d'une pseudo «mort» de la langue française se profilant à l'horizon, par le biais d'une «invasion» de la langue américaine. Il appartient naturellement aux commissions de terminologie chargées de convertir les termes anglo-américains en gallicismes de se pencher sur la question sémantique des concepts derrière les mots importés, au lieu d'inventer des lexies de nature hermétique pour un locuteur français. Par exemple (dans le domaine de l'informatique), au lieu de proposer «boule de commande», qui ne veut rien dire, il eût été plus simple de diffuser «bille», qui fait appel à la tradition populaire et s'inscrit dans le droit fil de la socio-terminologie, en ce que toute création de termes doit reposer sur un support sémantique familier aux locuteurs visés.

Il reste à faire un commentaire sur l'usage du français par les concepteurs de logiciels étudiés dans cette enquête. Ces équipes se sont en général bien comportées, si l'on exclut toutefois la transformation désinvolte de deux lexies anglaises en des termes bâtards : touche «off» et instruction «go». En effet, ce sont deux usages critiquables, dans la mesure où ces instructions ne semblent pas exister sur les claviers américains et, quant à la deuxième directive, elle ne se trouve même pas codifiée dans le «langage machine» ${ }^{24}$ où l'on trouve en revanche (en anglais), «GO TO» et non «GO» tout seul. Nous craignons détecter ici l'ignorance d'ingénieurs «s'adonnant» à leur péché mignon : imiter les Américains en articulant de l'anglais approximatif.

\section{RÉNOVER LA LANGUE FRANÇAISE}

Nous devrons donc à la fois calmer les appréhensions des puristes de la langue et rappeler aux scientifiques français que leur propre idiome comporte d'innombrables ressources dans son lexique et son génie créatif (témoin les revues spécialisées et les manuels théoriques étudiés dans cette enquête), dont il leur appartient de se servir s'ils veulent revendiquer toute appartenance à une communauté culturelle dans laquelle ils sont nés. Cela n'exclut pas la poursuite d'une connaissance (respectable) de l'anglais ou de toute autre langue étrangère pour communiquer à l'extérieur de la communauté francophone. Mais cela n'entraine pas le postulat que le français va s'affaiblir parce que l'on recourt à l'anglais pour communiquer à l'extérieur de cette grande communauté. Il est plus facile de (mal) parler l'anglais que de (bien) parler le français, c'est entendu, mais il n'y a rien de nouveau dans cette affirmation. En revanche, il est à la portée de tous les niveaux d'interlocuteurs francophones de 
puiser dans les ressources lexicales du français les moyens terminologiques existants, à créer ou à transformer, pour donner ${ }^{25}$ à un concept ou une lexie une allure de gallicisme. Il s'agit pour cela de renforcer l'enseignement du français.

Dès lors, il semblerait qu'une voie toute tracée apparaisse pour - essayons le mot — «remédier»au malaise linguistique des scientifiques français. $\mathrm{Ce}$ malaise est dû certainement à de l'ignorance scolaire $^{26}$, mais également à un engouement pour les idiomes américains - qui ne s'explique que parce que ressenti à distance. («L'herbe est toujours plus verte ailleurs», tandis que s'ils connaissaient les véritables paramètres de la société à laquelle ils aspirent, ces rêveurs risqueraient de déchanter - mais cela ressort d'un autre thème de recherche.) Un autre facteur contribuant pour une part importante à ce malaise semble provenir d'un pessimisme de mauvais aloi sur les chances de la science informatique française d'organiser des percées sur les marchés étrangers s'ils ne sont pas anglo-américains ${ }^{27}$; ce qui alimente le cercle vicieux du recours à la langue anglaise, alors que davantage de connaissances géopolitiques leur feraient saisir les opportunités commerciales de pays non seulement à culture francophone (Afrique, Asie du SudEst, Océanie, Caraïbes), mais également à culture francophile, tels certains États de l'ancienne Europe de l'Est.

Il serait peut être temps de rééduquer les scientifiques de l'Hexagone en leur prodiguant à nouveau un enseignement consacrant les valeurs lexicales du français, qui sont innombrables, au vu de l'histoire de cette langue. Le corollaire de cet axiome étant la nécessité de «rajeunir» la langue française en la dépouillant de ses éléments décoratifs, comme dirait $\mathrm{C}$. Hagège, pour ce qui est du domaine scientifique :

La valeur d'un article scientifique, si elle est fonction de sa clarté et de sa rigueur, n'est pas fonction du caractère littéraire de sa langue; le français ne peut à la fois prétendre élargir son audience dans le monde scientifique et conserver les traits d'un code de luxe dans les travaux de recherche à caractère technique ${ }^{28}$.

Cela fait appel à l'aménagement linguistique, inconcevable sans le recours aux industries de la langue. Celles-ci sont définies par P. Auger ${ }^{29}$ comme une discipline regroupant l'industrialisation de la langue (qui mesure l'efficacité des relations entre l'homme, la langue et la machine) et les technologies qu'elle met en œuvre. Il ajoute que «l'atteinte de cet objectif de développement de produits industriels de langue française est primordiale pour le maintien du statut international du français comme langue véhiculaire de la science et des techniques» ${ }^{30}$.

Cet objectif s'inscrit dans le cadre d'une philosophie certainement plus pragmatique que celle envisagée jusqu'à présent par l'éducation nationale en France et par le monde des lettres, toujours à l'affût de manquements aux règles de la langue française.

Il nous faut citer ici le prix Nobel (année 1991) de physique français P.-G. de Gennes ${ }^{31}$, qui plaide pour une réforme radicale de l'enseignement supérieur, en commençant par supprimer les grands concours, en orientant d'avantage la recherche vers les sciences appliquées que vers les maths pures, dont les Français sont friands, en rénovant le système de recrutement des professeurs et en accélérant la pression intellectuelle et compétitive sur le corps estudiantin, le but étant de délivrer un produit humain plus accordé avec les besoins de la société de demain, au lieu de survivre avec les vieux mythes des grands diplômes ouvrant les portes vers des emplois titulaires, dispensateurs de statut et de sécurité.

Redonner confiance aux locuteurs français sur le statut de leur langue est également un objectif majeur. Lorsque l'on entend dire par un responsable des ventes d'une entreprise de logiciel : «Edutainment est utilisé parce que nous n'avons pas trouvé que l'équivalent rende idée...», l'on se demande où cet individu a appris le français. En effet, il se trouve que l'équivalent français «logiciel d'éveil», fort clair, nous paraisse mieux traduire le propos de ce genre de logiciel pour enfants qu'edutainment, dont la facture phonétique est difficile à reproduire pour un francophone et qui est un ensemble abâtardi de deux mots anglais. Le fait que l'équivalent français s'exprime en deux mots au lieu d'un ne surcharge pas le discours et lui confère une clarté nécessaire à la compréhension du contexte, alors qu'un francophone est susceptible de percevoir Edutainment comme l'addition de deux mots : éducation et tènement(!), car c'est ce que l'on entend lorsque l'on prononce «Edutainment»

Il semblerait que les Français aient une fausse perception de leur langue. Citons sur ce plan M. Danzin, qui conseille le respect de l'orthographe traditionnelle pour permettre aux informaticiens de formuler leurs messages avec clarté. Il importe donc de refondre complètement l'impression que ces scientifiques ont de leur idiome. Ceci est l'un des messages que pourraient lancer les académiciens qui, au lieu de se pencher sur les questions secondaires d'emprunts à l'anglais, devraient attirer l'attention sur l'essentiel: faire du français une langue véhiculaire de la science grâce à la promotion sur le territoire hexagonal d'un certain créneau d'industries où les Français peuvent marquer des points, comme celle des télécommunications et, à présent, les industries de la langue.

Il est temps alors de rendre la langue de Descartes plus communicative ${ }^{32}$, encore qu'elle ait été jusqu'à présent tout à fait capable d'engendrer une terminologie correspondant aux concepts découverts par les chercheurs français, de même qu'elle est habile nous l'avons vu plus haut - à adapter les terminologies américaines au contexte culturel français. Mais il faut faire plus : d'abord se dépouiller de tout complexe face à l'anglais technique qui emprunte de toutes les façons la plupart de son vocabulaire savant au latin ou au grec, c'est-à-dire à un patrimoine auquel le français appartient et qui n'est certainement pas l'apanage de l'anglais; ensuite recourir au génie de la langue pour exploiter ses possibilités dérivatives, néologiques, etc., tout comme l'ont fait les écrivains sous revue, et enfin 
débarrasser l'expression scientifique française de tours alambiqués qui ne conviennent pas au message que cette branche de la connaissance vise à envoyer : clair, concis, à la grammaire impeccable et compris de tout le monde. Alors peut-être les Français se convaincront-ils que leur langue comporte des ressources qu'ils ne soupçonnaient pas. (Par exemple, exclure totalement le style déployé dans un article paru dans Meta sous le titre «The exquisite sophistication of French scholarly writing: French spirit or French letter?» ${ }^{33}$ ). Il est évident qu'une telle forme d'expression est la raison pour laquelle les scientifiques français auraient tendance à préférer l'anglais au français pour traduire leurs idées. Nous osons espérer que les perpétrateurs d'un tel morceau d'acrobatie linguistique en réalisent l'intransposabilité ${ }^{34}$ non seulement au langage courant, mais surtout scientifique. (Rappelons le propos de Pierre Lerat : «La vérité est que le technique n'est pas le sophistiqué, mais le réemployable, et que la culture linguistique la moins étriquée reste la plus portative dans les métiers des langues» ${ }^{35}$.)

En dernière analyse, un point majeur ne semble pas avoir été souligné par les études (en langue française) disponibles jusqu'à ce jour : l'influence du français de l'informatique sur l'anglais, pour la simple raison qu'il faut être chercheur aux États-Unis pour se rendre compte d'un tel phénomène, alors qu'en France l'on se trouve éloigné du mécanisme néologique américain.

Les seuls échos que les Français reçoivent de ce mécanisme semblent provenir essentiellement de contacts accidentels avec la langue technique, dus à des relations commerciales, soit directes, soit par le truchement de traductions, qui ne reflètent pas fidèlement, comme démontré plus haut, la valeur sémantique de l'anglais informatique. L'existence d'influences lexicales françaises sur l'anglais de l'informatique constitue un phénomène majeur dans le domaine de la terminologie, ainsi que l'a souligné Taylor, qui a publié dans Meta une étude sur les termes du vocabulaire à suffixe «ique» relevant de l'informatique et leur influence sur la langue anglaise ${ }^{36}$.

En un mot, qu'il s'agisse de ce dernier élément essentiel en matière de recherche terminologique ou des options pragmatiques proposées pour remédier à la situation de «malaise» linguistique des informaticiens français, du champ d'application de cette étude qui recouvre de manière synthétique des domaines aussi variés que la théorie, les logiciels, le jargon parlé ou journalistique des sciences d'information, de la création de «xéno-pérégrinismes» ou de la remise en cause de l'emprunt, cette recherche est susceptible d'apporter une nouvelle donne au débat qui se déroule actuellement, dans le sillage de la loi Toubon et autres inquiétudes linguistiques sévissant en France.

Dans le cadre de ce débat, et dans la mesure où l'on cessera de juger qu'un terme est français seulement s'il est attesté - selon une vieille habitude qui semble s'altérer avec l'influx d'une terminologie informatique française - la langue demeurera en bonne santé. Cette nouvelle flexibilité, prix de la survie d'une langue, nous permettra d'adhérer sans réserve aux propos de Julien Boithias, de l'Association de défense de la langue française ${ }^{37}$ :

En utilisant une expression un peu vulgaire mais bien française, je dirais donc qu'un mot est français lorsqu'il a une 'gueule' de mot français. De même qu'on reconnaît facilement qu'un mot a une 'gueule' de mot allemand ou de mot polonais, pourquoi n'en serait-il pas de même d'un mot français?

ÉLISABETH EEK

Université George-Mason, Fairfax, États-Unis

\section{Notes}

1. De Gourmont, R. (1899) : Esthétique de la langue française, Paris, Société du Mercure de France, cité par C. Hagège dans: Le français et les siècles, Paris, Éditions Odile Jacob, 1987, p. 86.

2. Enseignement qui devrait être rendu nécessaire dans tous les centres - universitaires ou non - d'instruction informatique pour, précisément, éviter les embûches sémantiques à l'origine de cet emprunt «paria». Voilà un agenda dont devraient se saisir les défenseurs de la langue pour le promouvoir au rang de programme éducatif à mettre en œuvre au plus tôt. Ceci pourrait renverser la tendance actuelle des emprunts tous azimuts à l'anglais informatique et opérer - à long terme - une reconversion de l'activité mentale des intéressés. Il sera en effet difficile de les rééduquer, prisonniers qu'ils sont d'une «américanomania» dénotant un flagrant manque de confiance en leur patrimoine culturel et donc en eux-mêmes.

3. Voir définition ci-dessous.

4. Voir l'interview du ministre François Bayrou parue dans Le Figaro Magazine du 11 septembre 1993, sous le titre «Le ministre au tableau».

5. Nous nous autoriserons ce néologisme approprié au contexte qui nous intéresse.

6. Car c'est précisément l'objectif de toute modernisation d'une société : promouvoir la recherche scientifique en s'assurant que la terminologie indigène «suit» en s'adaptant. Il ne s'agit pas en revanche de décourager les scientifiques français en diminuant la valeur de l'enseignement de leur langue. C'est pour cela que ces derniers se sont «raccrochés» récemment à l'anglais : parce qu'ils ont ressenti une impression de démission de la part des autorités éducatives nationales face à l'avalanche d'une terminologie anglo-américaine qui n'a pas peur de ses origines : multiples, sur les plans linguistiques, culturels et conceptuels. Cette langue est faite d'emprunts non seulement au français classique, au latin, au grec, aux racines germaniques, mais également à des lexies contemporaines, aussi bien françaises qu'autres. Il serait temps, donc, que les institutions éducatives et académiques en France se rendent compte de la véritable structure de l'anglo-américain pour faire adapter au français la même souplesse : une pâte à modeler faite de multiples apports utilisés sans 
vergogne - pourquoi en serait-il autrement ? — de la part de ses locuteurs.

7. Manuels et revues écrits, rappelons-le, par les théoriciens et journalistes de l'informatique et non par les praticiens, moins conscients du phénomène de langue que leurs collègues.

8. Ceux-ci, bien que ne s'apparentant pas en principe aux praticiens qui ne font que manipuler (et donc ne conçoivent pas intellectuellement) l'outil informatique, ont commis dans ces cas précis des erreurs sémantiques assimilables à celles des praticiens.

9. Dutourd, Jean (1995): «Petit plan de salut public», Défense de la langue française, $\mathrm{n}^{\circ} 175$, janvier-février-mars 1995 , p. 2.

10. Opinion également exprimée par J. Humbley, dans l'article «L'emprunt sémantique dans la terminologie de l'informatique», paru dans Meta, 32 (3), 1987, pp. 321-325.

11. Groupe de travail ayant étudié l'impact des arrêtés de terminologie sur l'informaticien français et dont les résultats ont paru dans la revue Terminologie et traduction, $\mathrm{n}^{\circ} 1,1991$.

12. Il s'agit ici du fabricant de systèmes informatiques - matériels et logiciels - n'incorporant pas de traduction dans ses produits, sachant que ceux-ci trouveront acheteurs en raison soit d'une supériorité technique authentique, soit d'une arrogance commerciale ignorante des contraintes linguistiques des acheteurs.

13. Considérant la tendance actuelle du marché.

14. Comme c'est le cas des magazines et logiciels qui ont été analysés par la suite.

15. Rappelons que cette étude s'applique à un manuel de théorie informatique : «SGBD relationnels» («Systèmes de gestion de bases de données relationnels»). Les polices utilisées dans cette démonstration sont l'exacte réplique de celles employées par l'auteur.

16. «Opérateur» de l'algèbre relationnelle permettan une combinaison de deux tables suivant deux attributs communs.

17. L'usage de l'indicatif dans cette circonstance est prévu par Grévisse, p. 1604, paragraphe 1071, a).

18. Système de gestion de bases de données.

19. Kahn, Philippe (1995) : «An open letter to Gates and Manzi», Wall Street Journal, 24 mars 1995, Eastern edition, p. A 14

20. Chansou, Michel (1981) : Recherche sur la planification linguistique en français contemporain, Thèse pour le doctorat de troisième cycle en linguistique, Université de Paris-Nord, p. 67.

21. Gaudin, François (1995) : «Champs, clôtures et domaines : des langues de spécialité à la culture scientifique», Meta, 40 (2), pp. 229-236.

22. Gaudin, François (1995) : «Entrevue avec Lionel Salem. Aventures et mésaventures d'un dictionnaire des sciences», Meta, 40 (2), pp. 320-329.

23. Une percée dans le domaine des supraconducteurs, mise au point par des chercheurs de Grenoble, Le Figaro du 5 octobre 1993, p. 13.

24. Anglicisme (machine language) dont la langue française peut fort bien s'accommoder, en raison précisément de la transparence des termes.
25. Lorsqu'il s'agit de faire appel, comme le reconnaît J. Humbley lui-même, au patrimoine gréco-latin (commun à l'anglais et au français) pour dénommer des concepts nouveaux. Voir note $\mathrm{n}^{\circ} 10$.

26. Dans la mesure où les acteurs de cette profession semblent avoir oublié les ressources lexicales (par exemple, le mécanisme de formation des dérivés, néologismes, etc.) de leur propre langue.

27. Cette attitude fut clairement démontrée dans les réponses fournies lors d'enquêtes téléphoniques conduites auprès de cadres d'entreprises de logiciel au cours des années 1993, 1994 et 1995.

28. Hagège, Claude (1987): Le français et les siècles, Paris, Éditions Odile Jacob, p. 282.

29. Auger, Pierre (1989): «Informatique et terminologie: revue des technologies nouvelles», Meta, 34 (3), pp. 485-492.

30. Voir note précédente.

31. Pierre Gilles de Gennes, «Études: 'Le Nobel français veut une révolution'», Le Figaro Magazine, 5 novembre 1994.

32. Nous incorporons sous ce vocable les propriétés de clarté, concision et simplicité caractérisant toute langue contemporaine scientifique, mais que l'on ne trouve pas dans un texte de Hawthorne ou Stendhal, par exemple.

33. Colson, Jacques (1993) : «The exquisite sophistication of French scholarly writing: French spirit or French letter?» Meta, 38 (3), pp. 426-439.

34. Au regard des néologismes relevés dans les textes étudiés, nous considérons ce dérivé tout à fait approprié au contexte de rajeunissement de la langue de «tous les jours».

35. Propos cité au dos de la couverture de Meta, 40 (2), juin 1995.

36. Taylor, Glen (1988): «INFOR-M-A-T-IQUE: A Study of "informatique"-related "ique" terms», Meta, 33 (4), pp. 550-560.

37. Boithias, Lucien : «Qu'est-ce qu'un mot français ?», Défense de la langue française, $\mathrm{n}^{\circ} 175$, janvier-février-mars 1995, pp. 12-13. 